\title{
Emergence of soft quark excitations by the coupling with a soft mode of the QCD critical point
}

\author{
Masakiyo Kitazawa, ${ }^{1}$ Teiji Kunihiro, ${ }^{2}$ and Yukio Nemoto $^{3}$ \\ ${ }^{1}$ Department of Physics, Osaka University, Toyonaka, Osaka 560-0043, Japan \\ ${ }^{2}$ Department of Physics, Kyoto University, Kyoto 606-8502, Japan \\ ${ }^{3}$ Department of Physiology, St. Marianna University School of Medicine, Kanagawa 216-8511, Japan
}

(Dated: May 26, 2022)

\begin{abstract}
We study the quark spectrum at nonzero temperature and density near the critical point (CP) of the chiral phase transition incorporating effects of the scalar- and pseudoscalar-density fluctuations in a chiral effective model with a nonzero current quark mass. It is known that the soft mode associated with the second-order transition at the CP lies in the spacelike region of the scalardensity fluctuation. We find that the soft mode influences the quark spectrum significantly near the $\mathrm{CP}$, resulting in the shift of the quasiquark peak. Effects of the composite stable pions on the quark spectrum near the $\mathrm{CP}$ are also discussed.
\end{abstract}

\section{INTRODUCTION}

The phases of quantum chromodynamics (QCD) at nonzero temperature $(T)$ and the quark-chemical potential $(\mu)$ are of much interest both theoretically and experimentally. It has been established in lattice QCD with the physical quark masses that there does not exist a genuine phase transition at nonzero temperature and zero baryon density but only a rapid crossover from the hadronic state to the quark-gluon plasma [1-4]. In the cold and dense region $\mu \gtrsim T$, in contrast, reliable lattice simulations are still missing because of the notorious sign problem, although various attempts have been made [59]. Low-energy effective models of QCD [10 12 tell us that there is a critical point $(\mathrm{CP})$ at $T>0$ and $\mu>0$ where the critical line of the first-order phase transition in the low-temperature region terminates and that becomes second order [13]. We mention that there can be variants or alternatives of the phase diagram with multiple or no critical points when the vector interaction [14], color superconductivity with and without charge neutrality and/or the anomaly terms are incorporated [15 20], even apart from the possibility of inhomogeneous phases [21, 22].

The global structure of the QCD phase diagram, including the identification of the $\mathrm{CP}$, can be investigated experimentally using relativistic heavy ion collisions by comparing the collision events with different collision energies. An experimental program to perform such an investigation, called the beam energy scan program, is now ongoing at the Relativistic Heavy Ion Collider [23]. The existence of the CP may be confirmed by the measurement of fluctuation observables in this program [24]. Experiments at the planned facilities at GSI, NICA and J-PARC would also contribute to reveal physical properties of the $\mathrm{CP}$ as well as the its existence.

We emphasize here that the CP appears when the chiral symmetry is explicitly broken, and the identifications of the order parameter of the second-order transition and the associated soft modes are not simple and involved [25, 26]. In the chiral limit where the current $u, d$ quarks are massless, the order parameter is the chiral condensate, and the critical line of the first-order transition in the low-temperature region is connected at the tricritical point (TCP) with the critical line of the second-order transition; the associated soft modes to the phase transition are the amplitude and phase fluctuations of the order parameter, which are identified with the sigma and the pionic modes, respectively [27]. We notice that there is no scalar-vector coupling at the TCP due to the chiral symmetry.

When the chiral symmetry is explicitly broken with the nonzero current quark masses, the second-order transition in the high-temperature region turns to a crossover and the sigma and pionic modes stay massive even at the CP: Indeed the sigma mode has a mass $m_{\sigma} \simeq 2 m$ around the $\mathrm{CP}$ with $m$ being the dynamically generated constituent quark mass. Moreover, in such a case with an explicit chiral-symmetry breaking at $\mu \neq 0$, the scalar-vector cross-correlation term $\left\langle:\left(\bar{\psi} \gamma^{0} \psi\right)(\bar{\psi} \psi):\right\rangle$ does not vanish, and, hence, the scalar mode is coupled with the density-density correlator $\left\langle:\left(\bar{\psi} \gamma^{0} \psi\right)^{2}:\right\rangle$ 14]; this is because charge conjugation symmetry is violated with nonzero $\mu$, and the left- and right-handed quarks are coupled owing to the breaking of chiral symmetry. It has been shown [25, 26] that the soft mode, the mass of which vanishes at the $\mathrm{CP}$, is a superposition of the numberdensity fluctuation (phonon) and the sigma mode, and the QCD CP belongs to the same universality class as the liquid-gas phase transition, which is called "model H" in the classification scheme by Hohenberg and Halperin 28]: Precisely speaking, the soft mode associated with the CP consists of not only the sigma and the phonon mode but also the entropy fluctuations, i.e., the hydrodynamic modes [25, 26].

In the present work, we investigate how the soft mode associated with the CP affects in turn the quark spectrum near the CP. We employ the simplest Nambu-JonaLasinio (NJL) model 10 12, 29] without the vector coupling to describe the soft mode associated with the CP. In this model, the collective mode in the scalar channel has a support in the spectral function not only in the 
timelike region but also in the spacelike region, the latter of which is composed of particle-hole excitations and describes the soft mode associated with the CP [25].

It is known that the quarks coupled with the bosonic excitations at nonzero temperature show an unexpected rich structure in the spectral function, even apart from the formation of the celebrated plasmino excitations at extremely high temperatures as given in the hardthermal-loop (HTL) approximation in gauge theories [30, 31]. In [32], the spectral properties of quarks coupled with the soft modes at zero density were examined above but near the critical temperature of the secondorder chiral transition in the chiral limit. It was found that a novel peak is formed around the zero energy as well as the normal and the antiplasmino ones, which then makes a three-peak structure in the quark spectral function: The formation of the far-low-lying peak is owed to the mixing between a quark (antiquark) and an antiquark hole (quark hole) by a resonant scattering of the quasiquarks with the soft modes with small but nonzero masses 32 34]. Such a formation of the three-peak structure in the spectral function of a fermion coupled with a bosonic excitation at $T \neq 0$ is now confirmed beyond one loop 35 39]. Furthermore, a similar third peak in the ultrasoft region has been shown to exist in gauge theories at high temperature beyond the HTL approximation on the basis of a novel resummation technique [40]. It is also shown that the neutrino spectrum shows similar three peaks containing an ultrasoft branch at electroweak scale temperature [41].

It should be noted, however, that such a three-peak structure in the fermion spectrum tends to be suppressed as the fermion mass becomes large [42]. Thus one might naturally suspect that the quark spectrum near the CP, which is realized when the chiral symmetry is explicitly broken, would not have any anomalous structure like that seen near the critical temperature in the chiral limit. However, a recent paper [43] of the present authors shows that it may not be the case: In fact, the quark spectral function in this region can have an anomalously low-lying peak due to the coupling with the stable pionic modes with a nonhyperbolic dispersion relation around the pseudocritical temperature, which leads to a van Hove singularity of the joint density of states in the imaginary part of the quark self energy.

In the present paper, we shall show that the quark spectrum around the CP shows yet another unexpected behavior due to the coupling to the phononlike soft mode in the spacelike region. The spectral properties of fermions at nonzero $\mu$ have been studied in gauge theories in the hard dense limit 31] and also the electron spectrum in high density plasma [44]. The modification of the quark spectrum near the $\mathrm{CP}$ is different from both of these cases, because the soft mode associated with the $\mathrm{CP}$ consists of particle-hole states in the spacelike region. It will also be shown that the effects of the van Hove singularity present around the crossover region at $\mu=0$ almost die out around the CP.

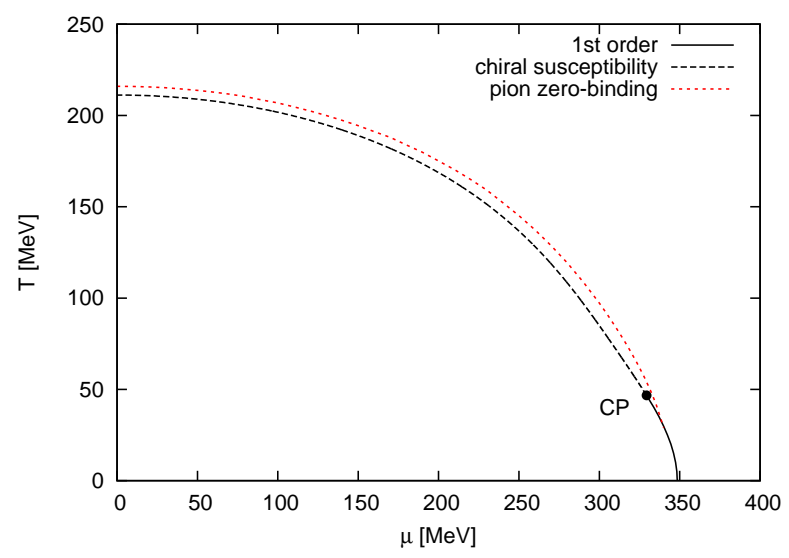

FIG. 1. The phase diagram of the chiral transition. CP means the critical point. The solid line denotes the first-order phase transition, the dashed line the maximum of the chiral susceptibility at each $\mu$. The dotted line denotes the pion zerobinding temperatures where the binding energy of the stable pionic modes vanishes.

The paper is organized as follows. In the next section, we introduce the NJL model to investigate the phase diagram of the chiral transition, and formulate the fluctuation modes and the quark self energy. We then discuss the properties of the fluctuation modes in Sec. III. The numerical results are shown in Sec. IV] A summary and concluding remarks are given in Sec. $\mathrm{V}$.

\section{FORMALISM}

In this section, after introducing the model used in this study we present the phase diagram obtained in this model. We then give the expressions of the meson and quark propagators in the random-phase approximation at nonzero $T$ and $\mu$. The calculation procedures described in Secs. IIB IIC are basically the same as those in our previous study [4], except for the introduction of nonzero quark chemical potential $\mu$.

\section{A. Model and phase diagram}

We employ the two-flavor NJL model [29]

$$
\mathcal{L}=\bar{\psi}\left(i \not \partial-m_{0}\right) \psi+G_{S}\left[(\bar{\psi} \psi)^{2}+\left(\bar{\psi} i \gamma_{5} \boldsymbol{\tau} \psi\right)^{2}\right],
$$

as an effective model of low-energy QCD [11], and study the effect of fluctuation modes in the scalar $(\sigma)$ and pseudoscalar $(\pi)$ channels on the spectral properties of quarks near the phase boundary, with $\tau$ being the flavor $\mathrm{SU}(2)$ Pauli matrices. The coupling constant $G_{S}=5.5 \mathrm{GeV}^{-2}$, the current $u, d$-quark mass $m_{0}=5.5 \mathrm{MeV}$, and the three-dimensional cutoff $\Lambda=631 \mathrm{MeV}$ are determined so as to reproduce the pion mass, the pion decay constant, and the quark condensate in vacuum [11]. 
As mentioned in Sec. I. a complete description of the effects of the specific collective modes on the quark spectrum necessitates the inclusion of the couplings of the number-density and the entropy fluctuations to the quark field. There are some attempts to do it: A model Lagrangian has been proposed to take into account the phonon-quark coupling in the meson-quark model [45]. We also note that although in a quite different context, Shen and Reddy [46] calculated the neutrino scattering from hydrodynamic modes in hot and dense neutron matter using a transport equation. However, an extension of the model for such a complete description of the hydrodynamic modes and their couplings to the quark is beyond the scope of the present work.

In Fig. 1, we show the phase diagram of the chiral transition in the mean-field approximation (MFA). There is a first-order transition at low temperature and large chemical potential region shown by the solid line, which terminates at the $\mathrm{CP}$, denoted by $\mathrm{CP}$ in the figure, with the critical temperature $T_{c}$ and the chemical potential $\mu_{c}$ given by

$$
T_{c} \simeq 47 \mathrm{MeV}, \quad \mu_{c} \simeq 329 \mathrm{MeV} .
$$

The phase transition is of second order at this point. The transition becomes crossover for $\mu<\mu_{c}$. For a guide of the phase boundary for $\mu<\mu_{c}$, the temperatures at which the chiral susceptibility has the maximum at a fixed chemical potential, i.e., the ridge of the chiral susceptibility, are shown by the dashed line.

\section{B. Fluctuation modes}

Next we construct the propagator of the scalar and pseudoscalar fluctuations at nonzero $T$ and $\mu$. The soft mode associated with the $\mathrm{CP}$ manifests itself in the scalar fluctuations. The pions, which are described by the pseudoscalar fluctuations, are still bound states near the CP in this model as shown in Sec. III] The excitation properties of these fluctuation modes are characterized by the spectral functions for the quark-antiquark excitations in the $\sigma$ and $\pi$ channels

$$
\rho_{\sigma(\pi)}\left(\boldsymbol{p}, p_{0}\right)=-\frac{1}{\pi} \operatorname{Im} D_{\sigma(\pi)}^{R}\left(\boldsymbol{p}, p_{0}\right),
$$

where $D_{\sigma}^{R}\left(\boldsymbol{p}, p_{0}\right)$ and $D_{\pi}^{R}\left(\boldsymbol{p}, p_{0}\right)$ are the retarded quarkantiquark propagators in each channel. The corresponding imaginary-time propagators in the random-phase approximation read

$$
\mathcal{D}_{\sigma(\pi)}\left(\boldsymbol{p}, \nu_{n}\right)=-\frac{1}{1 /\left(2 G_{S}\right)+\mathcal{Q}_{\sigma(\pi)}\left(\boldsymbol{p}, \nu_{n}\right)},
$$

where $\mathcal{Q}_{\sigma}\left(\boldsymbol{p}, \nu_{n}\right)$ and $\mathcal{Q}_{\pi}\left(\boldsymbol{p}, \nu_{n}\right)$ are the one-loop quarkantiquark polarization functions

$$
\begin{aligned}
\mathcal{Q}_{\sigma}\left(\boldsymbol{p}, \nu_{n}\right)= & T \sum_{m} \int \frac{d^{3} q}{(2 \pi)^{3}} \operatorname{Tr}\left[\mathcal{G}_{0}\left(\boldsymbol{q}, \omega_{m}\right)\right. \\
& \left.\times \mathcal{G}_{0}\left(\boldsymbol{p}+\boldsymbol{q}, \nu_{n}+\omega_{m}\right)\right], \\
\mathcal{Q}_{\pi}\left(\boldsymbol{p}, \nu_{n}\right)= & \frac{T}{3} \sum_{m} \int \frac{d^{3} q}{(2 \pi)^{3}} \operatorname{Tr}\left[i \gamma_{5} \boldsymbol{\tau} \mathcal{G}_{0}\left(\boldsymbol{q}, \omega_{m}\right)\right. \\
& \left.\times i \gamma_{5} \boldsymbol{\tau} \mathcal{G}_{0}\left(\boldsymbol{p}+\boldsymbol{q}, \nu_{n}+\omega_{m}\right)\right],
\end{aligned}
$$

with $\mathcal{G}_{0}\left(\boldsymbol{p}, \omega_{n}\right)=\left[\left(i \omega_{n}+\mu\right) \gamma_{0}-\boldsymbol{p} \cdot \vec{\gamma}-m\right]^{-1}$ being the quark propagator and $\nu_{n}=2 n \pi T$ and $\omega_{n}=(2 n+$ 1) $\pi T$ denoting the Matsubara frequencies for bosons and fermions, respectively. $m=m_{0}+m_{D}$ is the constituent quark mass where $m_{D}$ is the dynamically generated mass from spontaneous chiral-symmetry breaking evaluated in the MFA. After the summation of the Matsubara frequency and the analytic continuation with a replacement $i \nu_{n} \rightarrow p_{0}+i \eta$, we obtain the retarded functions $Q_{\sigma(\pi)}^{R}\left(\boldsymbol{p}, p_{0}\right)$ and $D_{\sigma(\pi)}^{R}\left(\boldsymbol{p}, p_{0}\right)$. For the numerical calculation of $Q_{\sigma(\pi)}^{R}\left(\boldsymbol{p}, p_{0}\right)$, we first calculate the imaginary part that is free from the ultraviolet divergence and then evaluate the real part with the Kramers-Kronig relation

$$
\operatorname{Re} Q_{\sigma(\pi)}^{R}\left(\boldsymbol{p}, p_{0}\right)=-\frac{1}{\pi} \mathrm{P} \int_{-\Lambda^{\prime}}^{\Lambda^{\prime}} d p_{0}^{\prime} \frac{\operatorname{Im} Q_{\sigma(\pi)}^{R}\left(\boldsymbol{p}, p_{0}^{\prime}\right)}{p_{0}-p_{0}^{\prime}},
$$

where $\mathrm{P}$ denotes the principal value and the energy cutoff $\Lambda^{\prime}=2 \sqrt{\Lambda^{2}+m^{2}}$ is determined such that $D_{\sigma}^{R}\left(\boldsymbol{p}, p_{0}\right)$ at $\boldsymbol{p}=0$ and $p_{0}=0$ in the spacelike region diverges at the CP determined by the MFA. This is a softening condition associated with the $\mathrm{CP}$ and is consistent with the introduction of the cutoff $\Lambda$ into the thermodynamic potential in the MFA.

The imaginary parts of $Q_{\sigma}^{R}\left(\boldsymbol{p}, p_{0}\right)$ and $Q_{\pi}^{R}\left(\boldsymbol{p}, p_{0}\right)$ are proportional to the difference between the decay and creation rates of each mode and take nonzero values for $\left|p_{0}\right|>E_{\mathrm{thr}}(p)$ and $\left|p_{0}\right|<p$, with $E_{\mathrm{thr}}(p)=\sqrt{p^{2}+4 m^{2}}$ and $p=|\boldsymbol{p}|$. The decay processes into a quark and an antiquark take place for $p_{0}>E_{\mathrm{thr}}(p)$, where the threshold energy $E_{\mathrm{thr}}(p)$ is the lowest value of the sum of excitation energy of a quark and an antiquark with a fixed total momentum $p$. The decay process in the spacelike region, $\left|p_{0}\right|<p$, is the Landau damping, which is the scattering processes of a quark or an antiquark with the fluctuation modes.

The propagators $D_{\sigma(\pi)}^{R}\left(\boldsymbol{p}, p_{0}\right)$ may have poles on the lower-half complex energy plane corresponding to collective excitations. In vacuum, $D_{\pi}^{R}\left(\boldsymbol{p}, p_{0}\right)$ has a boundstate pole on the real axis corresponding to the pseudoNambu-Goldstone pion [43]. The pole of $D_{\sigma}^{R}\left(\boldsymbol{p}, p_{0}\right)$, on the other hand, has an imaginary part, which implies that the sigma meson is a resonance state with a decay width.

When the poles of $D_{\pi}^{R}\left(\boldsymbol{p}, p_{0}\right)$ are located on the real axis, the dispersion relation of the stable pionic modes, 
$\omega_{\pi}(p)$, is obtained by solving the equation

$$
\begin{aligned}
{\left[\operatorname{Re} D_{\pi}^{R}\left(\boldsymbol{p}, \omega_{\pi}(p)\right)\right]^{-1} } & =-\frac{1}{2 G_{S}}-\operatorname{Re} Q_{\pi}^{R}\left(\boldsymbol{p}, \omega_{\pi}(p)\right) \\
& =0
\end{aligned}
$$

Because $\operatorname{Im} D_{\pi}^{R}\left(\boldsymbol{p}, p_{0}\right)$ vanishes for $p<\left|p_{0}\right|<E_{\mathrm{thr}}(p)$, Eq. (7) gives the correct dispersion relation only for $p<$ $\omega_{\pi}(p)<E_{\mathrm{thr}}(p)$. The residue $Z_{\pi}(p)$ of the bound pole is given by

$$
\begin{aligned}
\frac{1}{Z_{\pi}(p)} & =-\left.\frac{1}{\pi} \frac{\partial\left[D_{\pi}^{R}\left(\boldsymbol{p}, \omega_{\pi}\left(p_{0}\right)\right)\right]^{-1}}{\partial p_{0}}\right|_{p_{0}=\omega_{\pi}(p)} \\
& =-\left.\frac{1}{\pi} \frac{\partial Q_{\pi}^{R}\left(\boldsymbol{p}, \omega_{\pi}\left(p_{0}\right)\right)}{\partial p_{0}}\right|_{p_{0}=\omega_{\pi}(p)} .
\end{aligned}
$$

When Eq. (7) has a solution in the range of $p_{0}$ at which $\operatorname{Im} D_{\pi}^{R}\left(\boldsymbol{p}, p_{0}\right)$ takes a nonzero value, we refer to the solution as the quasipole.

\section{Quark self energy}

In this study, we consider the effect of the fluctuation modes in the $\sigma$ and $\pi$ channels near the CP on spectral properties of quarks. As a first step of such an analysis, we use the random-phase approximation [43], where the fluctuation modes are constructed by the undressed quarks and antiquarks in a non-self-consistent way. In addition, we limit our attention to the quark spectral function at zero momentum, since the soft mode influences the quark spectrum at vanishing momentum most strongly.

The quark self energy in the random-phase approximation for $p=0$ is given by

$$
\begin{aligned}
& \tilde{\Sigma}\left(\boldsymbol{p}=\mathbf{0}, \omega_{n}\right) \equiv \tilde{\Sigma}\left(\omega_{n}\right) \\
& =-T \sum_{m} \int \frac{d^{3} q}{(2 \pi)^{3}}\left[\mathcal{D}_{\sigma}\left(\boldsymbol{q}, \omega_{n}-\omega_{m}\right) \mathcal{G}_{0}\left(\boldsymbol{q}, \omega_{m}\right)\right. \\
& \left.\quad+3 \mathcal{D}_{\pi}\left(\boldsymbol{q}, \omega_{n}-\omega_{m}\right) i \gamma_{5} \mathcal{G}_{0}\left(\boldsymbol{q}, \omega_{m}\right) i \gamma_{5}\right],
\end{aligned}
$$

where the factor 3 in front of $\mathcal{D}_{\pi}$ comes from the isospin degeneracy.

After the summation of the Matsubara frequency in Eq. (9) and the analytic continuation, $i \omega_{n} \rightarrow p_{0}+i \eta$, we obtain the retarded quark self energy,

$$
\Sigma^{R}\left(p_{0}\right)=\Sigma_{\sigma}^{R}\left(p_{0}\right)+\Sigma_{\pi}^{R}\left(p_{0}\right)
$$

with

$$
\begin{aligned}
\Sigma_{\sigma}^{R}\left(p_{0}\right) & =\int \frac{d^{4} q}{(2 \pi)^{4}} \frac{\left(\gamma^{0}+m / E_{q}\right) \pi \rho_{\sigma}\left(\boldsymbol{q}, q_{0}\right)}{q_{0}-p_{0}+E_{q}-\mu-i \eta} \\
& \times\left[1+n\left(q_{0}\right)-f_{-}\left(E_{q}\right)\right] \\
& +\int \frac{d^{4} q}{(2 \pi)^{4}} \frac{\left(\gamma^{0}-m / E_{q}\right) \pi \rho_{\sigma}\left(\boldsymbol{q}, q_{0}\right)}{q_{0}-p_{0}-E_{q}-\mu-i \eta} \\
& \times\left[n\left(q_{0}\right)+f_{+}\left(E_{q}\right)\right]
\end{aligned}
$$

and

$$
\begin{aligned}
\Sigma_{\pi}^{R}\left(p_{0}\right) & =\int \frac{d^{4} q}{(2 \pi)^{4}} \frac{\left(\gamma^{0}-m / E_{q}\right) 3 \pi \rho_{\pi}\left(\boldsymbol{q}, q_{0}\right)}{q_{0}-p_{0}+E_{q}-\mu-i \eta} \\
& \times\left[1+n\left(q_{0}\right)-f_{-}\left(E_{q}\right)\right] \\
& +\int \frac{d^{4} q}{(2 \pi)^{4}} \frac{\left(\gamma^{0}+m / E_{q}\right) 3 \pi \rho_{\pi}\left(\boldsymbol{q}, q_{0}\right)}{q_{0}-p_{0}-E_{q}-\mu-i \eta} \\
& \times\left[n\left(q_{0}\right)+f_{+}\left(E_{q}\right)\right],
\end{aligned}
$$

and $E_{q}=\sqrt{\boldsymbol{q}^{2}+m^{2}}$. The functions $n(x)$ and $f_{ \pm}(x)$ are the Bose-Einstein and the Fermi-Dirac distribution functions, $n(x)=[\exp (x / T)-1]^{-1}$ and $f_{ \pm}(x)=[\exp ((x \pm$ $\mu) / T)+1]^{-1}$, respectively. For the evaluation of these equations, we first compute the imaginary parts for which the $q_{0}$ integral is performed analytically and the $q$ integral is carried out with the cutoff $\Lambda$, and then evaluate the real parts with a relation 32 .

$$
\operatorname{Re} \Sigma_{\sigma(\pi)}^{R}\left(p_{0}\right)=-\frac{1}{\pi} \mathrm{P} \int_{-\Lambda}^{\Lambda} d p_{0}^{\prime} \frac{\operatorname{Im} \Sigma_{\sigma(\pi)}^{R}\left(p_{0}^{\prime}\right)}{p_{0}-p_{0}^{\prime}}
$$

where the cutoff $\Lambda$ is the same as that used in Sec. IIB.

The retarded quark propagator for zero momentum reads

$$
G^{R}\left(p_{0}\right)=\frac{1}{\left(p_{0}+i \eta+\mu\right) \gamma^{0}-m-\Sigma^{R}\left(p_{0}\right)},
$$

which is decomposed into

$$
G^{R}\left(p_{0}\right)=G_{+}\left(p_{0}\right) \Lambda_{+} \gamma^{0}+G_{-}\left(p_{0}\right) \Lambda_{-} \gamma^{0}
$$

using the projection operators $\Lambda_{ \pm}=\left(1 \pm \gamma_{0}\right) / 2$. Here $G_{+}\left(G_{-}\right)$is the retarded quark (antiquark) propagator defined as

$$
G_{ \pm}\left(p_{0}\right)=\frac{1}{2} \operatorname{Tr}\left[G^{R} \gamma^{0} \Lambda_{ \pm}\right]=\frac{1}{p_{0} \mp m+\mu-\Sigma^{ \pm}\left(p_{0}\right)}
$$

with $\Sigma_{ \pm}\left(p_{0}\right)=\operatorname{Tr}\left[\Sigma^{R} \Lambda_{ \pm} \gamma^{0}\right] / 2$. The quasiquark $\left(\rho_{+}\right)$and quasiantiquark $\left(\rho_{-}\right)$spectral functions are then expressed with $G_{ \pm}$as

$$
\rho_{ \pm}\left(p_{0}\right)=-\frac{1}{\pi} \operatorname{Im} G_{ \pm}\left(p_{0}\right)
$$

It is noted that $p_{0}=0$ denotes the Fermi level at $T=0$, while $p_{0}+\mu=0$ is the Dirac level in our notation.

\section{SOFT MODE AND MESONIC MODES}

In this section, we summarize the behavior of the fluctuation modes at nonzero $T$ and $\mu$ as the basic ingredients for the analysis of the quark propagator.

Let us first focus on the scalar channel. Figure 2 shows the spectral function $\rho_{\sigma}\left(\boldsymbol{p}, p_{0}\right)$ at $T=48 \mathrm{MeV}$ and $\mu \simeq 328 \mathrm{MeV}$, which is on the ridge of the chiral susceptibility and very close to the $\mathrm{CP}$. We see that there is 
not only a peak in the timelike region corresponding to the sigma mode 47] but also a sharp peak in the spacelike region around $p \approx 0$ and $p_{0} \approx 0$, the latter of which is precisely the soft mode associated with the CP. The small width of the soft mode comes from the Landau damping that is effective in the spacelike region. The propagator $D_{\sigma}^{R}\left(\boldsymbol{p}, p_{0}\right)$ in the scalar channel has a pole associated with the soft mode: The pole moves toward and eventually reaches the origin in the complex energy plane as the system approaches the CP; i.e., both the mass and the width of the soft mode vanish at the CP. The nature of the soft mode is actually the collective particle-hole excitation familiar in many-body physics, which is possible owing to the presence of the density (or the Fermi sphere).

In contrast, the sigma mode in the timelike region has a nonzero mass and the width even at the $\mathrm{CP}$, the latter of which gradually broadens as the momentum increases. In Fig. 3, the peak position of the sigma mode, which is approximately regarded as the mass of the sigma mode, is plotted at the same $T$ and $\mu$ as those in Fig. 2

Next we turn to the pseudoscalar channel. As $T$ and/or $\mu$ are raised, the constituent quark mass $m$ becomes smaller while the rest mass of the pionic mode $\omega_{\pi}(0)$ becomes larger. Then, the pionic mode acquires a width since the decay into a quark and an antiquark pair becomes possible. We call the temperature at which the rest pionic mode becomes unstable as the pion zero-binding temperature $T_{\mathrm{ZB}}$, which is determined by

$$
\left[\operatorname{Re} D_{\pi}^{R}(\mathbf{0}, 2 m)\right]_{T=T_{\mathrm{ZB}}}^{-1}=0,
$$

for a given $\mu$. In Fig. 1. $T_{\mathrm{ZB}}$ as a function of $\mu$ is plotted with the dotted line. We see that $T_{\mathrm{ZB}}$ is always higher than the dashed line representing the temperature at which the chiral susceptibility has the maximum for a given $\mu$. It is also noted that the $\mathrm{CP}$ is located below $T_{\mathrm{ZB}}$ in our analysis, which means that the pionic modes are stable at the CP. As $\mu$ goes high, $T_{\mathrm{ZB}}$ decreases and eventually merges into the critical line of the first-order transition at $\mu \gtrsim 340 \mathrm{MeV}$.

The decay of a pionic mode into a quark and an antiquark pair is also possible when the dispersion relation $\omega_{\pi}(p)$ becomes larger than $E_{\mathrm{thr}}(p)$ even for $T<T_{\mathrm{ZB}}$, which implies that the pionic modes with a large velocity relative to the medium can be unstable owing to the medium effect. In Fig. 3, the dispersion relation $\omega_{\pi}(p)$ is also plotted; one sees that $\omega_{\pi}(p)$ becomes larger than $E_{\text {thr }}(p)$ at $p \simeq 575 \mathrm{MeV}$. This is due to the fact that the dispersion relation of the pionic modes is not the hyperbolic form, $\omega_{\pi}(p) \neq \sqrt{p^{2}+\omega_{\pi}^{2}(0)}$. In a previous study [43], the present authors showed that such an anomalous dispersion relation leads to a divergence of the joint density of states of the pionic modes and a quark, and gives rise to a van Hove singularity in the quark self energy. Although this is also the case near the $\mathrm{CP}$, it will be found that the singularity is negligibly weak.

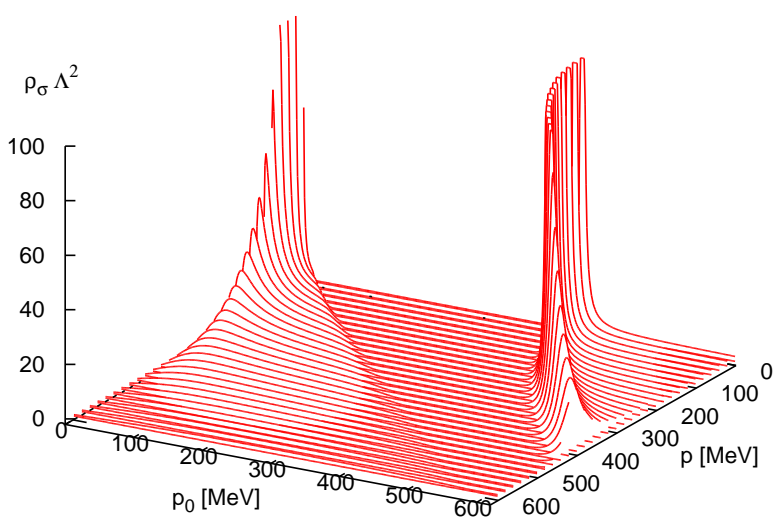

FIG. 2. The spectral function $\rho_{\sigma}\left(\boldsymbol{p}, p_{0}\right)$ at $T=48 \mathrm{MeV}$ and $\mu \simeq 328 \mathrm{MeV}$.

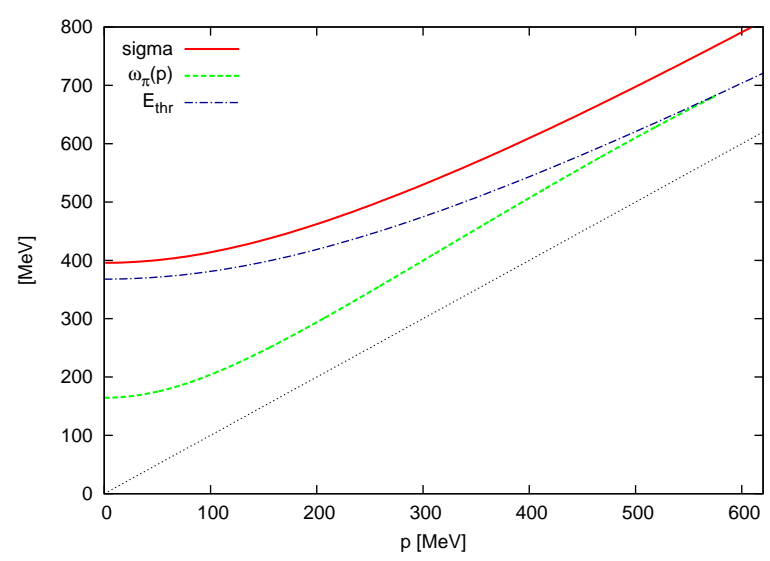

FIG. 3. The peak position of the sigma mode, the dispersion relation of the stable pionic mode, $\omega_{\pi}(p)$, and the threshold energy, $E_{\mathrm{thr}}=\sqrt{p^{2}+4 m^{2}}$, at $T=48 \mathrm{MeV}$ and $\mu \simeq 328$ $\mathrm{MeV}$.

\section{NUMERICAL RESULTS AND DISCUSSIONS}

In this section, we present the numerical results for the quark spectrum around the CP.

\section{A. Near the critical point}

We first see the quark spectral function $\rho_{+}\left(p_{0}\right)$ in

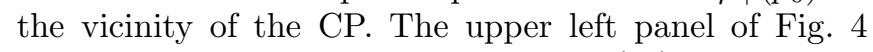
shows the quark spectral function $\rho_{+}\left(p_{0}\right)$ at $T=48$ $\mathrm{MeV}$ and $\mu \simeq 328 \mathrm{MeV}$, which is very close to the $\mathrm{CP}$ and on the ridge of the chiral susceptibility. We note that the quark spectral function in the MFA is given by $\rho_{+}\left(p_{0}\right)=\delta\left(p_{0}+\mu-m\right)$ with $m \simeq 184 \mathrm{MeV}$ being the corresponding constituent quark mass in the MFA. Fig- 

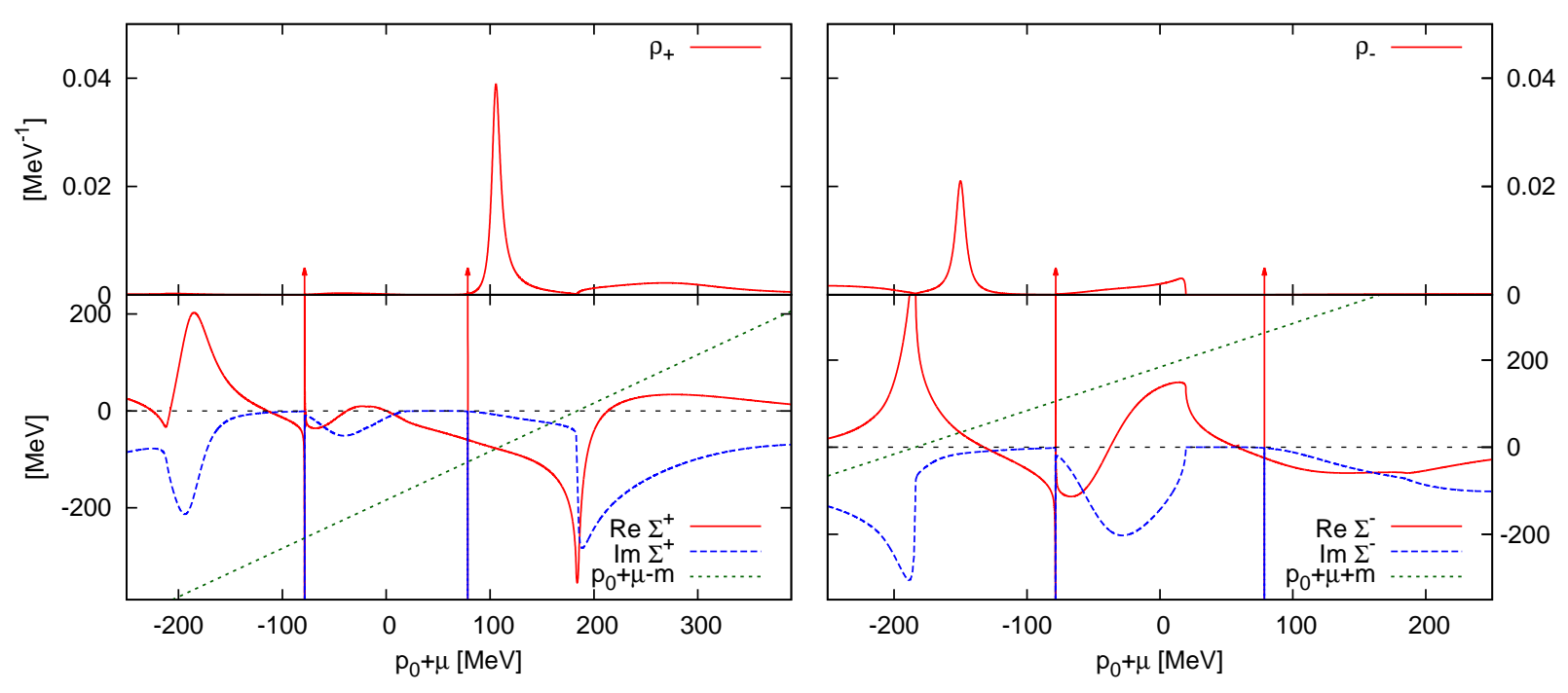

FIG. 4. The upper left (right) panel is the quark (antiquark) spectral function $\rho_{+}\left(p_{0}\right)\left(\rho_{-}\left(p_{0}\right)\right)$ for $T=48 \mathrm{MeV}$ and $\mu \simeq 328$ $\mathrm{MeV}$. Peaks with very small spectral weights $\left(Z \lesssim 10^{-2}\right)$ are shown in the arrows. The lower panels represent the real and imaginary parts of the corresponding quark self energies $\Sigma^{ \pm}\left(p_{0}\right)$. The dashed lines denotes $p_{0}+\mu \mp m$ with $m$ being the constituent quark mass.

ure 4 shows that the quark spectrum is largely modified from the one in the MFA; a peak given by the MFA at $p_{0}=m-\mu$ is totally absent. Moreover, one finds a prominent peak at an unexpectedly smaller energy than $m$, i.e., at $p_{0}+\mu \simeq 105 \mathrm{MeV}$; we note that the collective excitation corresponding to the peak at a positive energy has a positive quark number.

To figure out the origin of this anomalous peak, let us make a detailed analysis of the corresponding quark self energy. We show the real and imaginary parts of $\Sigma^{+}\left(p_{0}\right)$ in the lower left panel of Fig. 4. We see that there is a broad peak in $\operatorname{Im} \Sigma_{+}\left(p_{0}\right)$ around $p_{0}+\mu \simeq 190$ $\mathrm{MeV}$, implying that the single quark state with this energy decays into other states. Our numerical result with the decomposition Eqs. (10)-(12) shows that this peak of $\operatorname{Im} \Sigma^{+}\left(p_{0}\right)$ comes from the decay process of a quark into the soft mode shown in the left panel of Fig. 5. Because the strength of the soft mode is enhanced when both the energy and the momentum are close to zero in the spacelike region, this process is enhanced at $q \approx 0$ and $p_{0}+\mu-E_{q} \approx 0$. In addition, from Eq. (11), one sees that the Bose-Einstein distribution function in the $q$ integral in $\operatorname{Im} \Sigma^{+}\left(p_{0}\right)$ also acts to enhance the strength of the decay process around $p_{0}+\mu-E_{q}=0$.

The existence of a large peak in $\operatorname{Im} \Sigma^{+}\left(p_{0}\right)$ causes a steep rise in $\operatorname{Re} \Sigma^{+}\left(p_{0}\right)$ around it owing to the analytic property Eq. (13) which in turn gives rise to a zero in the real part of the inverse propagator for the quark (antiquark),

$$
p_{0}+\mu \mp m-\operatorname{Re} \Sigma^{ \pm}\left(p_{0}\right)=0 .
$$

When $\operatorname{Im} \Sigma^{ \pm}\left(p_{0}\right)$ is small at $p_{0}$ satisfying Eq. (19), the quark propagator has a peak there [32]. The solutions of Eq. (19) are graphically determined by points of inter-
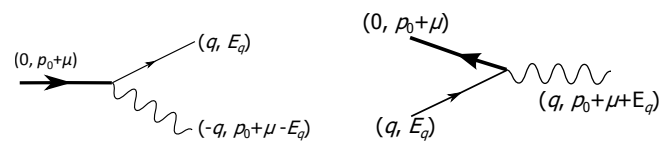

FIG. 5. The scattering processes that form the peaks in $\operatorname{Im} \Sigma_{+}$ at $p_{0}+\mu \simeq 190 \mathrm{MeV}$ (left) and $\operatorname{Im} \Sigma_{-}$at $p_{0}+\mu \simeq-190 \mathrm{MeV}$ (right). The thick solid line represents the quasiquark with $\left(\mathbf{0}, p_{0}+\mu\right)$, the thin solid line the on-shell free quark, and the wavy line the soft mode. The inverse processes are also possible.

section between $\operatorname{Re} \Sigma^{+}\left(p_{0}\right)$ and a line $p_{0}+\mu-m$ that is shown by a dashed green line in the lower left panel of Fig. 4. One sees that $\operatorname{Re} \Sigma^{+}\left(p_{0}\right)$ shows a peaklike structure around the peak in $\operatorname{Im} \Sigma^{+}\left(p_{0}\right)$ at $p_{0}+\mu \simeq 190 \mathrm{MeV}$. Consequently, this bending behavior of $\operatorname{Re} \Sigma^{+}\left(p_{0}\right)$ causes the shift of the position of the quasipole from that of the pole in the MFA. For $190 \lesssim p_{0}+\mu \lesssim 300 \mathrm{MeV}$, there is no clear peak but a bumplike structure in $\rho_{+}\left(p_{0}\right)$, because $\left|\operatorname{Im} \Sigma^{+}\left(p_{0}\right)\right|$ keeps large values and there is no quasipole.

We remark that the arrows at $p_{0}+\mu \simeq \pm 80 \mathrm{MeV}$ in the upper left panel of Fig. 4 indicate two sharp peaks in $\rho_{+}\left(p_{0}\right)$ with a vanishingly narrow width. The spectral weights of these peaks, however, are negligibly small. In fact, by defining the spectral weight of the peak by

$$
Z=\int_{\Delta} d p_{0} \rho_{ \pm}\left(p_{0}\right)
$$

where $\Delta$ is a range of $p_{0}$ that well covers the peak, one gets $Z \lesssim 10^{-2}$ for these peaks, while the sum of the spectral weight is $\int d p_{0} \rho_{+}\left(p_{0}\right)=1$ in our definition. Despite the sharpness of the peak structure, therefore, these peaks do not have physical significance. 


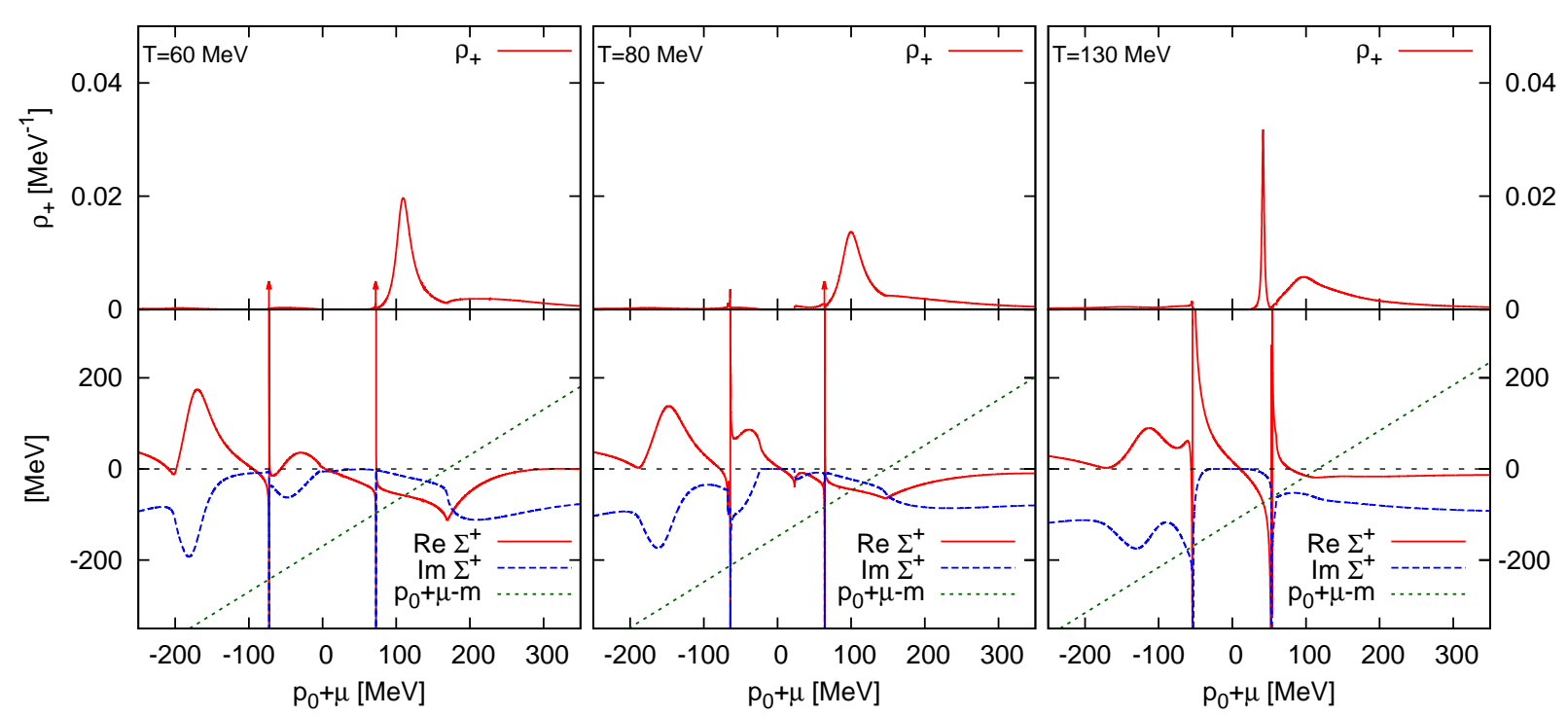

FIG. 6. The same as Fig. 4 but at $T=60 \mathrm{MeV}$ and $\mu \simeq 320 \mathrm{MeV}$ (left), $T=80 \mathrm{MeV}$ and $\mu \simeq 304 \mathrm{MeV}$ (middle), and $T=130 \mathrm{MeV}$ and $\mu \simeq 258 \mathrm{MeV}$ (right). These are on the ridge of the chiral susceptibility.

The emergence of these peaks is understood by the van Hove singularity triggered by the nonhyperbolic dispersion relation of the stable pionic modes $\omega_{\pi}(p)$ [43]. As discussed in Sec. III, $\omega_{\pi}(p)$ has a nonhyperbolic form near the $\mathrm{CP}$ owing to the violation of Lorentz symmetry in medium. In Ref. [43], it is discussed that such a distorted dispersion relation can give rise to zeros of the relative velocity between the quark and the bound pion, and hence the divergences of the joint density of states $\left[d\left\{E_{q}-\omega_{\pi}(q)\right\} / d q\right]^{-1}$ at some energies. Reflecting these divergences, $\Sigma^{+}\left(p_{0}\right)$ and $\rho_{+}\left(p_{0}\right)$ also diverge at these energies as the van Hove singularity.

In Ref. [43], we have shown that the van Hove singularity can significantly alter the spectral properties of quarks near the pseudocritical temperature at $\mu=0$. While the same singularity manifests itself even near the $\mathrm{CP}$, the strength of the singularity is weak. Therefore, the singularity near the $\mathrm{CP}$ would easily be blurred by the effects neglected in the present analysis, such as the contribution of higher-order terms of the quark self energy, in contrast to the case discussed in Ref. [43]. We thus do not discuss this structure in more detail in the present study.

The spectral function for the antiquark sector $\rho_{-}\left(p_{0}\right)$ for the same $T$ and $\mu$ is shown in the upper right panel of Fig. 4. One sees a peak at $p_{0}+\mu \simeq-145 \mathrm{MeV}$ whose position is shifted from that in the MFA, $-m=-184 \mathrm{MeV}$. To identify the origin of the shift, we show $\operatorname{Im} \Sigma^{-}\left(p_{0}\right)$ in the lower right panel of Fig. 4. One finds a peak in $\operatorname{Im} \Sigma^{-}\left(p_{0}\right)$ at $p_{0}+\mu \simeq-190 \mathrm{MeV}$. Our numerical analysis shows that this peak corresponds to a scattering process of an antiquark and a quark into the soft mode as denoted in the right panel of Fig. [5. Then, similarly to the previous discussion on $\rho_{+}\left(p_{0}\right)$, the peak distorts $\operatorname{Re} \Sigma^{-}\left(p_{0}\right)$ and gives rise to a new quasipole at $p_{0}+\mu \simeq-190$
$\mathrm{MeV}$. In this way, the antiquark sector also receives considerable modification from the soft mode near the CP. In $\rho_{-}\left(p_{0}\right)$, we also find peaks with vanishingly narrow widths as denoted by arrows in the upper right panel. The spectral strengths of these quasipoles are both negligible, $Z \lesssim 10^{-2}$.

\section{B. The case away from the critical point}

In this section, we examine how the spectral properties of the quark are affected by the soft mode associated with the $\mathrm{CP}$ when the system is away from the $\mathrm{CP}$; we show $\rho_{+}\left(p_{0}\right)$ for some combination of $(T, \mu)$ with $T>T_{c}$ and $\mu<\mu_{c}$. Although we only show $\rho_{+}\left(p_{0}\right)$, the spectral properties of the antiquark is qualitatively the same as those of the quark.

We first see the quark spectrum by varying $T$ and $\mu$ along the ridge of the chiral susceptibility. The quark spectral functions $\rho_{+}\left(p_{0}\right)$ and the self energies $\Sigma^{+}\left(p_{0}\right)$ on the ridge are shown in Fig. [ for $T=60,80$, and 130 $\mathrm{MeV}$; the corresponding chemical potentials are $\mu \simeq 320$, 304 , and $258 \mathrm{MeV}$, respectively. Behaviors of $\rho_{+}\left(p_{0}\right)$ and $\Sigma^{+}\left(p_{0}\right)$ for $T=60 \mathrm{MeV}$ shown in the far-left panels are

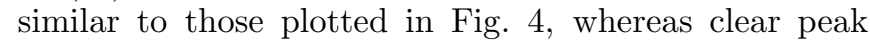
structures in these functions, i.e., the peaks in $\rho_{+}\left(p_{0}\right)$ at $p_{0}+\mu \simeq 105 \mathrm{MeV}$ and $\operatorname{Im} \Sigma^{+}\left(p_{0}\right)$ at $p_{0}+\mu \simeq 190$ $\mathrm{MeV}$, observed near the CP are slightly obscured for this $T$. This result shows that the soft mode associated with the $\mathrm{CP}$ can affect the quark spectrum even apart from the $\mathrm{CP}$ on the ridge of the chiral susceptibility. As $T$ is raised further, the effect of the soft mode gradually ceases to exist as shown in the middle panels of Fig. 6. In the lower middle panel one finds that the bump structure at $p_{0}+\mu \simeq 190 \mathrm{MeV}$ almost disappears for $T=80$ 
$\mathrm{MeV}$. Accordingly, the energy shift of the position of the quasiquark peak compared with the MFA value becomes smaller as shown in the upper middle panel. The lower right panel shows that the bump structure in $\operatorname{Im} \Sigma^{+}\left(p_{0}\right)$ disappears almost completely for $T=130 \mathrm{MeV}$.

In the upper right panel of Fig. 6, one finds that $\rho_{+}\left(p_{0}\right)$ again forms a sharp peak at $p_{0}+\mu$ significantly smaller than the constituent quark mass $m$, although the effect of the soft mode associated with the CP is suppressed. From the behavior of $\Sigma^{+}\left(p_{0}\right)$ shown in the lower right panel, one finds that this modification comes from the singular behavior of $\Sigma^{+}\left(p_{0}\right)$ around $p_{0}+\mu \simeq 50 \mathrm{MeV}$. As discussed in Ref. [43], these singularities are the van Hove singularity in the scattering process of quarks and pionic modes induced by the nonhyperbolic dispersion relation of the pionic modes. This result shows that the effect of the pionic modes becomes more prominent as $\mu$ is lowered instead of the soft mode associated with the CP.

Off the ridge of the chiral susceptibility, the strength of the soft mode rapidly decreases as $T$ and/or $\mu$ are away from the CP. The strength of the peak in the quark spectrum originated from the scattering of the soft mode accordingly decreases rapidly. For example, when $T$ is raised from the $\mathrm{CP}$ with fixed $\mu=\mu_{c}$, the distortion of the quark spectrum due to the soft mode is insignificant already at $T \simeq 1.05 T_{c}$.

\section{BRIEF SUMMARY AND CONCLUDING REMARKS}

In this paper, we have investigated the quark spectrum at nonzero temperature and density near the $\mathrm{CP}$ of the chiral transition in an effective model. The soft mode associated with the second-order transition at the $\mathrm{CP}$ is the scalar-density fluctuation in the spacelike region [25]. In the present study, we have investigated the effect of this soft mode on the quark spectrum for the first time.
We have shown that the soft mode strongly couples to a quark and an antiquark at vanishing momentum near the CP. As a result of this coupling, a quasiquark peak is created at the energy significantly lower than that in the mean-field approximation. It is also found that the strong modification of the quark spectrum due to the soft mode is observed over a wide range of $T$ and $\mu$ on the ridge of the chiral susceptibility. The effect of the soft mode, however, is suppressed rapidly off the ridge. In our previous study [43], we found that the quark spectrum near the pseudocritical temperature at $\mu=0$ is strongly modified by the van Hove singularity [43]. Although such a singularity is observed even for nonzero $\mu$, the effect of the singularity is negligibly weak near the CP.

In the present study, we have limited our attention to the quark spectral function at zero momentum, since the soft mode influences the quark spectrum at vanishing momentum most strongly. On the other hand, excitation modes near the Fermi momentum are relevant degrees of freedom in high density but low-temperature systems. The study of modes near the Fermi surface is left as a future work. As mentioned in Sec. IIB a complete analysis of the quark spectrum near the $\mathrm{CP}$ will require us to incorporate all the hydrodynamic modes including the number-density fluctuation (phonon) and entropy fluctuations. The coupling to the density fluctuations may be taken into account by the inclusion of the vector interaction [14] within this model. There are some attempts to incorporate the fermion-hydrodynamic modes coupling in the analysis of the phase structure and transport properties of the many body systems [45, 46]. We hope that we can report on such an analysis that extensively takes care of the coupling of the hydrodynamic modes to the quark near the $\mathrm{CP}$ elsewhere.

This work was supported by JSPS KAKENHI Grant No. 25800148, No. 24340054, and No. 24540271. T.K. was partially supported by the Core Stage Back UP program in Kyoto Univeristy, and by the Yukawa International Program for Quark-hadron Sciences.
[1] C. Bernard et al. [MILC Collaboration], Phys. Rev. D 71, 034504 (2005) hep-lat/0405029.

[2] M. Cheng et al., Phys. Rev. D 74, 054507 (2006) hep-lat/0608013.

[3] Y. Aoki, G. Endrodi, Z. Fodor, S. D. Katz and K. K. Szabo, Nature 443, 675 (2006) hep-lat/0611014.

[4] S. Ejiri et al., Phys. Rev. D 80, 094505 (2009) arXiv:0909.5122 [hep-lat]].

[5] Z. Fodor and S. D. Katz, JHEP 0404, 050 (2004) hep-lat/0402006.

[6] R. V. Gavai and S. Gupta, Phys. Rev. D 71, 114014 (2005) hep-lat/0412035.

[7] S. Ejiri, Eur. Phys. J. A 49, 86 (2013) arXiv:1306.0295 [hep-lat]].

[8] G. Aarts, L. Bongiovanni, E. Seiler, D. Sexty and I. -O. Stamatescu, Eur. Phys. J. A 49, 89 (2013) arXiv:1303.6425 [hep-lat]].
[9] O. Philipsen, Acta Phys. Polon. Supp. 5, 825 (2012) arXiv:1111.5370 [hep-ph]].

[10] S. P. Klevansky, Rev. Mod. Phys. 64, 649 (1992).

[11] T. Hatsuda and T. Kunihiro, Phys. Rept. 247, 221 (1994) arXiv:hep-ph/9401310.

[12] M. Buballa, Phys. Rept. 407, $205 \quad$ (2005) hep-ph/0402234.

[13] M. Asakawa and K. Yazaki, Nucl. Phys. A 504, 668 (1989).

[14] T. Kunihiro, Phys. Lett. B 271 (1991) 395.

[15] M. Kitazawa, T. Koide, T. Kunihiro and Y. Nemoto, Prog. Theor. Phys. 108, 929 (2002) hep-ph/0207255, hep-ph/0307278.

[16] Z. Zhang, K. Fukushima and T. Kunihiro, Phys. Rev. D 79, 014004 (2009) arXiv:0808.0927 [hep-ph]]; Z. Zhang and T. Kunihiro, Phys. Rev. D 80, 014015 (2009) arXiv:0904.1062 [hep-ph]]. 
[17] T. Hatsuda, M. Tachibana, N. Yamamoto and G. Baym, Phys. Rev. Lett. 97, 122001 (2006) hep-ph/0605018; N. Yamamoto, M. Tachibana, T. Hatsuda and G. Baym, Phys. Rev. D 76, 074001 (2007) arXiv:0704.2654 [hep$\mathrm{ph}]$.

[18] P. de Forcrand and O. Philipsen, JHEP 0701, 077 (2007) hep-lat/0607017; JHEP 0811, 012 (2008) arXiv:0808.1096 [hep-lat]]; O. Philipsen, Prog. Theor. Phys. Suppl. 174, 206 (2008) arXiv:0808.0672 [hepph]]; K. Fukushima, Phys. Rev. D 78, 114019 (2008) arXiv:0809.3080 [hep-ph]]; J. W. Chen, K. Fukushima, H. Kohyama, K. Ohnishi and U. Raha, Phys. Rev. D 80, 054012 (2009) arXiv:0901.2407 [hep-ph]].

[19] H. Basler and M. Buballa, Phys. Rev. D 82, 094004 (2010) arXiv:1007.5198 [hep-ph].

[20] Z. Zhang and T. Kunihiro, Phys. Rev. D 83, 114003 (2011) arXiv:1102.3263 [hep-ph]].

[21] E. Nakano and T. Tatsumi, Phys. Rev. D 71, 114006 (2005) hep-ph/0411350.

[22] D. Nickel, Phys. Rev. Lett. 103, 072301 (2009) arXiv:0902.1778 [hep-ph]]; Phys. Rev. D 80, 074025 (2009) arXiv:0906.5295 [hep-ph]].

[23] M. M. Aggarwal et al. [STAR Collaboration], Phys. Rev. Lett. 105, 022302 (2010) arXiv:1004.4959 [nuclex]]; L. Adamczyk et al. [STAR Collaboration], Phys. Rev. Lett. 112, no. 3, 032302 (2014) arXiv:1309.5681 [nucl-ex]].

[24] M. A. Stephanov, K. Rajagopal and E. V. Shuryak, Phys. Rev. Lett. 81, 4816 (1998) hep-ph/9806219; Phys. Rev. D 60, 114028 (1999) hep-ph/9903292; M. Asakawa, S. Ejiri and M. Kitazawa, Phys. Rev. Lett. 103, 262301 (2009) arXiv:0904.2089 [nucl-th]]; M. Kitazawa, M. Asakawa and H. Ono, Phys. Lett. B 728, 386 (2014) arXiv:1307.2978.

[25] H. Fujii, Phys. Rev. D 67, 094018 (2003) arXiv:hep-ph/0302167; H. Fujii and M. Ohtani, Phys. Rev. D 70, 014016 (2004) arXiv:hep-ph/0402263.

[26] D. T. Son and M. A. Stephanov, Phys. Rev. D 70, 056001 (2004) hep-ph/0401052.

[27] T. Hatsuda and T. Kunihiro, Phys. Lett. B 145, 7 (1984); Phys. Rev. Lett. 55, 158 (1985).

[28] P. C. Hohenberg and B. I. Halperin, Rev. Mod. Phys. 49, 435 (1977).

[29] Y. Nambu and G. Jona-Lasinio, Phys. Rev. 122, 345
(1961); Phys. Rev. 124, 246 (1961).

[30] J. Frenkel and J. C. Taylor, Nucl. Phys. B 334, 199 (1990); E. Braaten and R. D. Pisarski, Nucl. Phys. B 339, 310 (1990); H. A. Weldon, Phys. Rev. D 26, 2789 (1982); Phys. Rev. D 40, 2410 (1989).

[31] M. Le Bellac, Thermal Field Theory, (Cambridge University Press, Cambridge, 1996).

[32] M. Kitazawa, T. Kunihiro and Y. Nemoto, Phys. Lett. B 633, 269 (2006) arXiv:hep-ph/0510167.

[33] M. Kitazawa, T. Kunihiro and Y. Nemoto, Prog. Theor. Phys. 117, 103 (2007) arXiv:hep-ph/0609164.

[34] D. Satow, Y. Hidaka and T. Kunihiro, Phys. Rev. D 83, 045017 (2011) arXiv:1011.6452 [hep-ph]].

[35] M. Harada, Y. Nemoto and S. Yoshimoto, Prog. Theor. Phys. 119, 117 (2008) arXiv:0708.3351 [hep-ph]].

[36] M. Harada and Y. Nemoto, Phys. Rev. D 78, 014004 (2008) arXiv:0803.3257 [hep-ph]].

[37] S. -x. Qin, L. Chang, Y. -x. Liu and C. D. Roberts, Phys. Rev. D 84, 014017 (2011) arXiv:1010.4231 [nuclth]]; A. Bashir, L. Chang, I. C. Cloet, B. El-Bennich, Y. -X. Liu, C. D. Roberts and P. C. Tandy, Commun. Theor. Phys. 58, 79 (2012) arXiv:1201.3366 [nucl-th]].

[38] H. Nakkagawa, H. Yokota and K. Yoshida, Phys. Rev. D 85, 031902 (2012) arXiv:1111.0117[hep-ph]]; Phys. Rev. D 86, 096007 (2012) arXiv:1208.6386.

[39] S. -x. Qin and D. H. Rischke, Phys. Rev. D 88, 056007 (2013) arXiv:1304.6547 [nucl-th]].

[40] Y. Hidaka, D. Satow and T. Kunihiro, Nucl. Phys. A 876, 93 (2012) arXiv:1111.5015 [hep-ph]].

[41] K. Miura, Y. Hidaka, D. Satow and T. Kunihiro, Phys. Rev. D 88, 065024 (2013) arXiv:1306.1701 [hep-ph]].

[42] M. Kitazawa, T. Kunihiro, K. Mitsutani and Y. Nemoto, Phys. Rev. D 77, 045034 (2008) arXiv:0710.5809 [hep$\mathrm{ph}]$.

[43] M. Kitazawa, T. Kunihiro and Y. Nemoto, Phys. Rev. D 89, 056002 (2014) arXiv:1312.3022 [hep-ph]].

[44] L. Hedin and S. Lundqvist, Solid State Phys. 23, 1 (1970).

[45] K. Kamikado, T. Kunihiro, K. Morita and A. Ohnishi, PTEP 2013, 053D01 (2013) arXiv:1210.8347 [hep-ph]].

[46] G. Shen and S. Reddy, arXiv:1311.6096 [nucl-th].

[47] T. Kunihiro, M. Kitazawa and Y. Nemoto, Proc. Sci. CPOD07 (2007) 041, arXiv:0711.4429 